\title{
Solution to Polynomial Equations, a New Approach
}

\author{
Fleur T. Tehrani \\ School of Engineering and Computer Science, California State University, Fullerton, CA, USA \\ Email: ftehrani@fullerton.edu
}

How to cite this paper: Tehrani, F.T. (2020) Solution to Polynomial Equations, a New Approach. Applied Mathematics, 11, 53-66.

https://doi.org/10.4236/am.2020.112006

Received: January 2, 2020

Accepted: January 18, 2020

Published: January 21, 2020

Copyright (c) 2020 by author(s) and Scientific Research Publishing Inc. This work is licensed under the Creative Commons Attribution International License (CC BY 4.0).

http://creativecommons.org/licenses/by/4.0/

\begin{abstract}
A new approach for solving polynomial equations is presented in this study. Two techniques for solving quartic equations are described that are based on a new method which was recently developed for solving cubic equations. Higher order polynomial equations are solved by using a new and efficient algorithmic technique. The proposed methods rely on initially identifying the vicinities of the roots and do not require the use of complicated formulas, roots of complex numbers, or application of graphs. It is proposed that under the stated conditions, the methods presented provide efficient techniques to find the roots of polynomial equations.
\end{abstract}

\section{Keywords}

Polynomial Solutions, Quartic Equations, Computer Algorithms

\section{Introduction}

Various methods have been developed to solve polynomial equations (e.g. [1] [2]) and many authors have presented techniques to estimate the bound of the roots and the number of the real roots of a polynomial (e.g. [3]-[9]). Starting with cubic equations, despite that formulas for the roots of the equations have existed for many years, using those formulas requires taking the roots of complex numbers repeatedly which introduces significant approximations into the results. Therefore, the efficient technique to solve cubic equations has been considered to be the use of numerical algorithms. A new method for solving cubic equations was introduced in 2016 [10] that did not require the roots of complex numbers, use of complicated formulas, or graphs. By using this method, the vicinity of a real root is initially identified and then the roots of the cubic equation are found efficiently even by hand calculation within minutes. The purpose of this paper is to introduce a new approach for solving polynomial equations of order 4 and 
higher. For solving quartic equations that have many applications in science and technology, two options are discussed. Both options are based on the recent method presented to solve cubic equations [10]. The first option can be used to find the roots of any quartic equation while the second option can be applied if the roots of the quartic equation are not all complex. By using these options, any quartic equation with real coefficients can be solved efficiently. This paper further presents a more general approach for solving higher order polynomial equations that can be programmed as an efficient algorithm on a computer. The proposed techniques rely on initially finding the vicinities of the individual roots and proceeding to finding the roots by using algebraic and/or iterative procedures. By estimating the vicinities of the individual roots, the methods are designed to be efficient and stable. Unlike many previous techniques, the presented methods do not involve the use of complicated formulas, roots of complex numbers, or graphs. This research is structured by finding efficient techniques to solve quartic equations and proceeds to development of a general method to solve high order polynomial equations. In the next section, the methods of solving quartic equations are discussed. The section is then followed by description of a method for solving polynomial equations of order 5 or higher. Examples of applications of the proposed techniques are presented to show the effectiveness of the methods.

\section{Theory}

\subsection{Quartic Equations}

Many techniques have been proposed for solving quartic equations (e.g. [11] [12] [13] [14] [15]). In this paper, two methods for solving quartic equations are described. In the first method, the quartic equation is reduced to a cubic as first described by Descartes [16], and then the cubic equation is solved by using the method discussed in [10]. In the second method, the single variable quartic equation is differentiated and the resulting cubic equation is solved by using the method in [10]. Then the roots of the quartic equation are found by using the roots of the derivative cubic equation. The two methods are described as follows.

\subsubsection{Method \#1 for Solving Quartic Equations}

A quartic equation can be written as:

$$
f(x)=x^{4}+\alpha_{1} x^{3}+\alpha_{2} x^{2}+\alpha_{3} x+\alpha_{4}=0
$$

If $x=z-\left(\alpha_{1} / 4\right)$, the above equation can be expressed in terms of $z$ as a depressed quartic equation:

$$
f(z)=z^{4}+a z^{2}+b z+c
$$

where:

$$
\begin{gathered}
a=\alpha_{2}-3\left(\alpha_{1}^{2} / 8\right) \\
b=\alpha_{3}-\left(\alpha_{1} \alpha_{2} / 2\right)+\left(\alpha_{1}^{3} / 8\right)
\end{gathered}
$$




$$
c=\alpha_{4}-\left(\alpha_{1} \alpha_{3} / 4\right)+\left(\alpha_{1}^{2} \alpha_{2} / 16\right)-3\left(\alpha_{1}^{4} / 256\right)
$$

Equation (2) can be factored into two quadratic equations as found originally by Descartes [16].

If $R_{1}$ and $R_{2}$ are two roots of this quartic equation:

$$
f(z)=\left(z-R_{1}\right)\left(z-R_{2}\right)\left(z^{2}+p z+q\right)=\left[z^{2}-\left(R_{1}+R_{2}\right) z+R_{1} R_{2}\right]\left[z^{2}+p z+q\right]
$$

If $R_{1}+R_{2}=\gamma$ and $R_{1} R_{2}=\lambda$, comparing this equation with Equation (2) yields:

$$
p=R_{1}+R_{2}=\gamma
$$

and:

$$
\begin{gathered}
a=q+\lambda-\gamma^{2} \\
b=\gamma(\lambda-q) \\
c=q \lambda
\end{gathered}
$$

Rearranging Equations (3) and (4) and taking the resulting equations to power 2:

$$
\begin{gathered}
\left(a+\gamma^{2}\right)^{2}=(\lambda+q)^{2} \\
(b / \gamma)^{2}=(\lambda-q)^{2}
\end{gathered}
$$

Subtracting Equation (7) from (6) and substituting $q \lambda$ by $c$ from Equation (5):

$$
\left(a+\gamma^{2}\right)^{2}-\left(b^{2} / \gamma^{2}\right)=4 c
$$

Multiplying this equation by $\gamma^{2}$ yields:

$$
\gamma^{6}+2 a \gamma^{4}+\left(a^{2}-4 c\right) \gamma^{2}-b^{2}=0
$$

If $\gamma^{2}=t$, Equation (9) can be re-written as:

$$
f(t)=t^{3}+\delta_{1} t^{2}+\delta_{2} t+\delta_{3}=0
$$

where:

$$
\begin{gathered}
\delta_{1}=2 a \\
\delta_{2}=a^{2}-4 c \\
\delta_{3}=-b^{2}
\end{gathered}
$$

The cubic Equation (10) has at least one real root $t_{1}$. That root can be found by using the method described in [10]. That method is not described here for brevity. Since $t=\gamma^{2}$, substituting $t_{1}$ in Equation (3):

$$
a+t_{1}=q+\lambda
$$

and $q=c \lambda$ from Equation (5). Therefore:

$$
a+t_{1}=\lambda+(c / \lambda) \text {, hence: }
$$

$$
\lambda^{2}-\left(a+t_{1}\right) \lambda+c=0
$$

which can be solved for $\lambda$. 
After having found $t_{1}$ and finding $\lambda=R_{1} R_{2}$ :

$$
\begin{gathered}
R_{1}+R_{2}=\sqrt{t_{1}} \\
R_{1} R_{2}=\lambda
\end{gathered}
$$

These equations can be solved for $R_{1}$ and $R_{2}$ which are two roots of Equation (2). The remaining roots of that equation, $R_{3}$, and $R_{4}$, can be found by solving:

$$
z^{2}+t_{1}^{0.5} z+(c / \lambda)=0
$$

Then the roots of Equation (1) can be found by subtracting $\alpha_{1} / 4$ from the values of the roots of $f(z)$.

\subsubsection{Method \#2 for Solving Quartic Equations}

This method can be used to solve quartic equations with real roots. The minimum and maximum points of the quartic function can be found by differentiating $f(x)$ in Equation (1) with respect to $x$ and setting the resulting equation to zero:

$$
g(x)=[\mathrm{d} f(x) / \mathrm{d} x] / 4=x^{3}+0.75 \alpha_{1} x^{2}+0.5 \alpha_{2} x+0.25 \alpha_{3}=0
$$

The roots of this cubic equation can be found by using the method described in [10]. The roots of $f(x)$ can then be found based on the roots of $g(x)$. However, the root types of $f(x)$ need to be determined first.

\section{1) Determining the root types}

Theorem 1. The quartic equation with real coefficients $f(x)$ has real roots if $\alpha_{4}<0$.

Proof: If $\alpha_{4}<0$, then at $x=0, f(x)=\alpha_{4}<0$

$$
\lim _{x \rightarrow \pm \infty} f(x)=x^{4}=+\infty
$$

$f(x)$ is a continuous function of $x$ on $(-\infty,+\infty)$. Therefore, for an $0<x_{1}<\infty$, $f\left(x_{1}\right)=0$ and $x_{1} \in R$ where $R$ denotes the set of real numbers.

Theorem 2. If $f(x)>0$ at its minimum points, $f(x)$ has no real root.

Proof: $f(x)>0$ at its minimum points, and:

$$
\lim _{x \rightarrow \pm \infty} f(x)=x^{4}=+\infty
$$

There fore, $f(x)>0$ for all $x \in R$.

Theorem 3. If $f(x)$ has one minimum point, $x_{\min }$, and $f\left(x_{\min }\right)<0, f(x)$ has two real roots.

Proof: $x_{\min } \in R, f\left(x_{\min }\right)<0$ and:

$$
\lim _{x \rightarrow \pm \infty} f(x)=x^{4}=+\infty
$$

Therefore for a continuous function, $f(x)$, there is an $x_{1} \in R$, and $-\infty<x_{1}<x_{\min }$, so that $f\left(x_{1}\right)=0$, and there is an $x_{2} \in R$, and $x_{\min }<x_{2}<+\infty$, so that $f\left(x_{2}\right)=0$.

Theorem 4. If $x_{1 \min }, x_{2 \min }$, and $x_{3 \max }$ are three real roots of $g(x)$ as denoted above, where $x_{1 \min }<x_{3 \max }<x_{2 \min }$, and $f(x)<0$ at $x_{1 \min }$, and at $x_{2 \min }$, and $f(x)>0$ at $x_{3 \max }$, all the roots of $f(x)$ are real. 
Proof:

$$
\begin{gathered}
f\left(x_{1 \min }\right)<0, \quad f\left(x_{3 \max }\right)>0 \text {, and } f\left(x_{2 \min }\right)<0 \text { and } \\
\lim _{x \rightarrow \pm \infty} f(x)=x^{4}=+\infty
\end{gathered}
$$

$f(x)$ is a continuous function of $x$ on $(-\infty,+\infty)$.

Therefore,

there is an $x_{1} \in R$, and $-\infty<x_{1}<x_{1 \min }$, so that $f\left(x_{1}\right)=0$,

there is an $x_{2} \in R$, and $x_{1 \min }<x_{2}<x_{3 \max }$, so that $f\left(x_{2}\right)=0$,

there is an $x_{3} \in R$, and $x_{3 \max }<x_{3}<x_{2 \min }$, so that $f\left(x_{3}\right)=0$,

there is an $x_{4} \in R$, and $x_{2 \min }<x_{4}<+\infty$, so that $f\left(x_{4}\right)=0$.

Table 1 shows the conditions that need to be checked to determine the root types of $f(x)$.

After determining the type of the roots of $f(x)$, the general procedure of finding the real roots can be followed as explained below.

\section{2) Procedure}

a) If $f(x)$ has two real roots:

i) If $\alpha_{4}>0$, one real root of $f(x)$ is between a minimum point and $x=0$. The point $x=0$ and the minimum point can be chosen as an upper and a lower limit for the real root $r_{1}$ respectively. Successive iterations can be used to find $r_{1}$ in that vicinity. $f(x)$ is then divided by $\left(x-r_{1}\right)$ and the procedure described in [10] can be used to find the remaining roots of the quartic equation.

ii) If $\alpha_{4}<0$, a line joining a minimum point to $x=0$ can be formulated and the intersection of that line with the horizontal axis may be chosen as an upper limit of the root if $f(x)$ is positive at that point. That point and the minimum point can be chosen as an upper and a lower limit of the real root $r_{1}$ respectively. Successive iterations can be used to find $r_{1}$ in that vicinity. $f(x)$ will then be divided by $\left(x-r_{1}\right)$ and the procedure described in [10] can be used to find the remaining roots of the quartic equation.

b) If $f(x)$ has four real roots:

iii) The maximum and the first minimum points can be chosen as an upper and a lower limit of one real root respectively. Successive iterations can be used to find that root. Similarly, the maximum and the second minimum point can be used as an upper and a lower limit for another real root. Successive iterations can be used to find that root. Then $f(x)$ can be divided by $\left[x^{2}-\left(r_{1}+r_{2}\right) x+r_{1} r_{2}\right]$ where $r_{1}$ and $r_{2}$ are the two determined real roots, and the remaining roots of the resulting quadratic equation can be found after the factorization.

\subsection{Method for Solving High Order Polynomial Equations}

An nth order single variable polynomial equation with real coefficients can be written as:

$$
f(x)=x^{n}+\alpha_{1} x^{n-1}+\alpha_{2} x^{n-2}+\alpha_{3} x^{n-3}+\cdots+\alpha_{n}=0
$$

where $\alpha_{1}, \cdots, \alpha_{n}$ are real numbers.

If the order of the equation is odd, it is bound to have one or more real roots. 
If $\mathrm{n}$ is even and $\alpha_{n}<0$, the polynomial has real roots. If $n$ is even and $\alpha_{n}>0$, the polynomial may or may not have real roots.

The value of $f(x)$ can be found at $0,-1$, and +1 to determine whether it has any roots at these points. If such roots are found, they can be separated by factorization. The value of $f(x)$ can also be found in the close vicinity of -1 and +1 (e.g. at $-1.1,-0.9,0,+0.9$, and +1.1 , etc.) to see if there are sign changes in $f(x)$. If there are, then successive iterations can be used to find a real root. Then by factorization that root can be separated, and focus can be made on roots whose magnitudes are considerably less than or greater than 1 .

Then for a root $r$, two conditions can hold:

1) If $|r|<1^{1}$

For a high order polynomial, if a root's magnitude is significantly less than 1 , as the order is increased, the polynomial will function as a subset of a Levi-Civita field [18]. By ignoring the high order terms of the polynomial:

$$
f(x) \approx \alpha_{n-2} r^{2}+\alpha_{n-1} r+\alpha_{n}
$$

Setting this equation to zero, its roots are:

$$
r=\left[-\alpha_{n-1} \pm s_{1}\right] / 2 \alpha_{n-2}
$$

where $s_{1}=\left[k_{1}\right]^{0.5}$ and $k_{1}=\left(\alpha_{n-1}^{2}-4 \alpha_{n-2} \alpha_{n}\right)$, and in order for $r$ to be real, $k_{1} \geq 0$.

As a rough estimate, if the second order term in Equation (15) can be ignored, $r \approx-\alpha_{n} / \alpha_{n-1}$.

Then it can be concluded that $\left|\alpha_{n} / \alpha_{n-1}\right|$ should be less than 1 .

If this condition holds, and $k_{1} \geq 0$, the values of $f(x)$ at the limits given by Equation (16) and also at $x=-\left(\alpha_{n} / \alpha_{n-1}\right)$ can be checked. If at two of the points, $f(x)$ has opposite signs, a real root between the points can be found by successive iterations. If $k_{1}<0$, but $\left|\alpha_{n} / \alpha_{n-1}\right|<1$, the value of the first derivative of $f(x),\left(f^{\prime}(x)\right)$ can be found at $\left(-\alpha_{n} / \alpha_{n-1}\right)$, and a line formulated at this point with the slope at the point given by $f^{\prime}(x)$. At the intersection of that line with the $x$-axis, $f(x)$ can be checked. If $f(x)$ at this point and $f(x)$ at $\left(-\alpha_{n} / \alpha_{n-1}\right)$ have opposite signs, a real root between the two points can be found.

2) If $|r|>1$

For a high order polynomial $(n \geq 5)$ :

$$
f(x) \approx r^{n}+\alpha_{1} r^{n-1}+\alpha_{2} r^{n-2}=r^{n-2}\left(r^{2}+\alpha_{1} r+\alpha_{2}\right)
$$

The non-zero roots of this equation are at:

$$
r=\left[-\alpha_{1} \pm s_{2}\right] / 2
$$

where $s_{2}=\left[k_{2}\right]^{0.5}$ and $k_{2}=\alpha_{1}^{2}-4 \alpha_{2}$, and in order for $\mathrm{r}$ to be real, $k_{2} \geq 0$.

If the lowest order of $n$ in Equation (17) is ignored, as a rough approximation, $r \approx-\alpha_{1}$ which means that under the conditions, $\left|\alpha_{1}\right|$ should be greater than 1. If this condition holds and $k_{2} \geq 0, f(x)$ can be found at the limits given by Equation (18) and also at $x=-\alpha_{1}$. If $f(x)$ at two of the points has opposite signs, a ${ }^{1}$ The number of the real roots for $|r|<1$ may be found by using other techniques [17]. 
real root between the points can be found by successive iterations. If $k_{2}<0$, but $\left|\alpha_{1}\right|>1, f^{\prime}(x)$ at $x=-\alpha_{1}$ can be found and a line formulated at $x=-\alpha_{1}$ with a slope given by $f^{\prime}(x)$ at that point. If the intersection of that line with the $x$-axis and the point $x=-\alpha_{1}$ produce $f(x)$ with opposite signs, a real root between the points can be found by successive iterations.

\section{Procedure}

The procedure of finding the real roots of an nth order single variable polynomial with real coefficients and $n \geq 5$ can be summarized as follows:

- Consider:

- $f(x)=x^{n}+\alpha_{1} x^{n-1}+\alpha_{2} x^{n-2}+\alpha_{3} x^{n-3}+\cdots+\alpha_{n}=0$.

- Determine $f(x)$ at $0,-1,+1$. If there is a root, separate it by factorization.

- Determine $f(x)$ in the vicinity of $|r|=1$ (e.g. $-1.1,-0.9,+0.9$, and +1.1 ). If there are sign changes in that region, use successive iterations between two points giving opposite signs for $f(x)$ to find a real root and separate that root by factorization.

Is $\left|\alpha_{n} / \alpha_{n-1}\right|<1$ ?

- If so, calculate $k_{1}=\left(\alpha_{n-1}^{2}-4 \alpha_{n-2} \alpha_{n}\right)$.

- Is $k_{1} \geq 0$ ?

- If $k_{1}>0$, find $f(x)$ at:

- $r=\left[-\alpha_{n-1} \pm s_{1}\right] / 2 \alpha_{n-2}$.

- where $s_{1}=\left[k_{1}\right]^{0.5}$,

- Calculate $f(x)$ at $x=\left(-\alpha_{n} / \alpha_{n-1}\right)$.

- If at two of these points, $f(x)$ has opposite signs, find a real root between those points by successive iterations (if $k_{1}=0$, use the points $-\alpha_{n-1} / 2 \alpha_{n-2}$, and $\left.-\alpha_{n} / \alpha_{n-1}\right)$.

- If $k_{1}<0$, find $f^{\prime}(x)$ at $\left(-\alpha_{n} / \alpha_{n-1}\right)$ and call it $m_{1}$. Then find:

- $x_{1}=\left(-\alpha_{n} / \alpha_{n-1}\right)$.

- $f\left(x_{1}\right)=y_{1}$.

- $x_{2}=x_{1}-\left(y_{1} / m_{1}\right)$.

- Find $f\left(x_{2}\right)$. If $f\left(x_{1}\right)$ and $f\left(x_{2}\right)$ have opposite signs, find a real root between the points by successive iterations and separate it by factorization.

Is $\left|\alpha_{1}\right|>1$ ?

- If so, calculate $k_{2}=\alpha_{1}^{2}-4 \alpha_{2}$.

- Is $k_{2} \geq 0$ ?

- If $k_{2}>0$, calculate $f(x)$ at $r=\left[-\alpha_{1} \pm s_{2}\right] / 2$.

- where $s_{2}=\left[k_{2}\right]^{0.5}$,

- Calculate $f(x)$ at $x=-\alpha_{1}$.

- If $f(x)$ at two of these points has opposite signs, find a real root between those points by successive iterations and separate it by factorization (if $k_{2}=0$, use $-\alpha_{1}$ and $-\alpha_{1} / 2$ as two points).

- If $k_{2}<0$,

- Find $f^{\prime}(x)$ at $x=-\alpha_{1}$ and call it $m_{2}$.

- $x_{3}=-\alpha_{1}$. 
- $f\left(x_{3}\right)=y_{3}$.

- $x_{4}=x_{3}-\left(y_{3} / m_{2}\right)$.

- Calculate $f\left(x_{4}\right)$. If $f\left(x_{3}\right)$ and $f\left(x_{4}\right)$ have opposite signs, find a real root between $X_{3}$ and $x_{4}$ by successive iterations and separate it by factorization.

If $\left|\alpha_{n} / \alpha_{n-1}\right|>1$ and $\left|\alpha_{1}\right|<1$, focus should be made on finding roots in the vicinity of $|r|=1^{2}$.

When after factorizations the order of the polynomial is reduced to 4 , use the procedure described in the preceding section to find the remaining roots.

\section{Examples}

\section{Example 1}

Assume:

$$
f(x)=x^{5}-8 x^{4}-21 x^{3}+8 x^{2}-4 x+240=0
$$

Therefore, $\alpha_{1}=-8, \alpha_{2}=-21, \alpha_{3}=8, \alpha_{4}=-4, \alpha_{5}=240$.

It is quickly determined that the polynomial does not have a root at $-1,0$, and +1 .

$$
\begin{gathered}
\left|\alpha_{n} / \alpha_{n-1}\right|=240 / 4=60>1 \\
\left|\alpha_{1}\right|=8>1 \\
k_{2}=\alpha_{1}^{2}-4 \alpha_{2}=8^{2}-4 \times(-21)=148>0 \\
s_{2}=\left[k_{2}\right]^{0.5}=12.16
\end{gathered}
$$

Limits of a root $=\left[-\alpha_{1} \pm s_{2}\right] / 2=-2.08,10.08$

$$
\begin{gathered}
f(-2.08)=283.23 \\
f(10.08)=978.12 \\
f\left(-\alpha_{1}\right)=f(8)=-10032
\end{gathered}
$$

Starting successive iterations with the points at 8 and 10.08 yields $r=10$ after 7 iterations.

By factorization:

$$
f_{1}(x)=f(x) /(x-10)=x^{4}+2 x^{3}-x^{2}-2 x-24
$$

To find the roots of the quartic function $f_{1}(x)$, one of the methods of solving quartic equations that were described above can be used. If method $\# 1$ is used:

$$
x=z-(2 / 4)=z-0.5
$$

and $f_{1}(z)=z^{4}-2.5 z^{2}-23.4375$.

The roots of $f_{1}(z)$ are found at $z= \pm 2.5$ and the roots of $f_{1}(x)$ are found as:

$$
r_{2}=-2.5-0.5=-3
$$

${ }^{2}$ In cases where some of the coefficients are very small compared with others, the terms associated with those small coefficients can be ignored in the estimation of the root vicinities. In those cases, focus can be made on the $1^{\text {st }}$ three remaining terms with highest orders for roots with large magnitudes, and on the last three remaining terms with lowest orders for roots with magnitudes significantly less than 1 . 


$$
r_{3}=2.5-0.5=2
$$

Then by factorization:

$$
f_{1}(x) /(x-2)(x+3)=x^{2}+x+4
$$

Setting: $f_{2}(x)=x^{2}+x+4=0$, yields:

$$
r_{4}, r_{5}=-0.5 \pm 1.936 i
$$

Therefore, the roots of the $5^{\text {th }}$ order polynomial $f(x)$ are found at: $-3,2,10$, and $(-0.5 \pm 1.936 i)$.

Figure 1 shows a graphical representation of $f(x)$.

\section{Example 2}

Assume:

$$
f(x)=x^{6}-2.5 x^{5}-24 x^{4}+8.5 x^{3}+38 x^{2}+126 x-72
$$

Therefore: $\alpha_{1}=-2.5, \alpha_{2}=-24, \alpha_{3}=8.5, \alpha_{4}=38, \alpha_{5}=126, \alpha_{6}=-72$. It is determined that $f(x)$ has no root at $-1,0$, and 1 .

Following the general procedure as described above:

$$
\begin{gathered}
\left|\alpha_{n} / \alpha_{n-1}\right|=72 / 126=0.57<1 \\
k_{1}=\left(\alpha_{n-1}^{2}-4 \alpha_{n-2} \alpha_{n}\right)=(126)^{2}-4 \times 38 \times(-72)=26820 \\
s_{1}=[26820]^{0.5}=163.768
\end{gathered}
$$

The limits for a root are calculated as:

$$
\left[-\alpha_{n-1} \pm s_{1}\right] / 2 \alpha_{n-2}=[-126 \pm 163.768] / 2 \times 38=-3.81,0.497
$$

and:

$$
\begin{gathered}
f(-3.81)=-461.9 \\
f(0.497)=-0.473 \\
f(0.57)=11.09
\end{gathered}
$$

A root of $f(x)$ is found between 0.497 and 0.57 at $r_{1}=0.5$ after 4 iterations.

Following the procedure to find the other roots:

$$
\begin{gathered}
\left|\alpha_{1}\right|=2.5>1 \\
k_{2}=\alpha_{1}^{2}-4 \alpha_{2}=(2.5)^{2}-4 \times(-24)=102.25 \\
s_{2}=\left[k_{2}\right]^{0.5}=10.11
\end{gathered}
$$

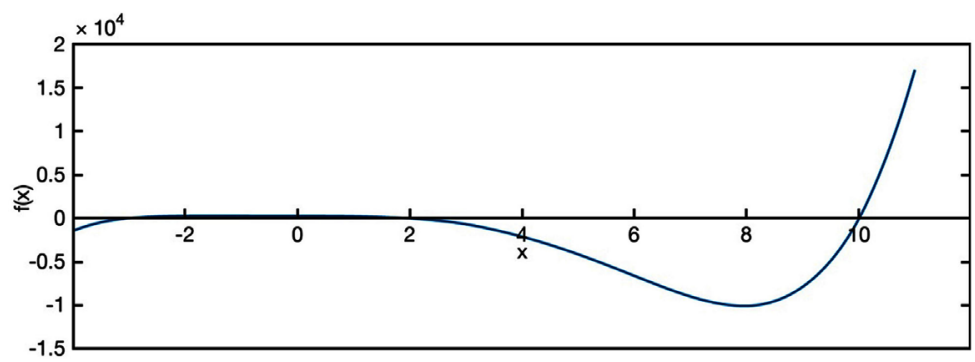

Figure 1. A graph representing $f(x)$ of example 1. 
Limits of a root $=\left[-\alpha_{1} \pm s_{2}\right] / 2=-3.8,6.3$

$$
\begin{gathered}
f(2.5)=-324.18 \\
f(-3.8)=-481 \\
f(6.3)=4261
\end{gathered}
$$

The iterations can start with the points at 2.5 and 6.3. After 8 iterations, a root is found at $r_{2}=6$.

By factorization:

$$
f(x) /(x-0.5)(x-6)=x^{4}+4 x^{3}-x^{2}-10 x-24
$$

The remaining roots are the roots of the quartic function:

$$
f_{1}(x)=x^{4}+4 x^{3}-x^{2}-10 x-24
$$

Following the procedure of method \#2 for solving quartic equations as described above, the roots of this quartic function are found at $-4,2$, and $(-1 \pm$ $1.4141)$. Therefore the roots of the $6^{\text {th }}$ order polynomial $f(x)$ are found at: $-4,0.5$, 2,6 and $(-1 \pm 1.414 i)$.

Figure 2 shows a graphical representation of $f(x)$.

\section{Example 3}

Assume:

$$
f(x)=x^{6}+0.8 x^{5}+2.1 x^{4}-1.5 x^{3}+3.1 x^{2}+4 x-5.1
$$

Therefore, $\alpha_{1}=0.8, \alpha_{2}=2.1, \alpha_{3}=-1.5, \alpha_{4}=3.1, \alpha_{5}=4, \alpha_{6}=-5.1$.

It can be seen that $\left|\alpha_{1}\right|<1$, and $\left|\alpha_{6} / \alpha_{5}\right|=1.275>1$. Therefore, focus should be made on finding roots with magnitudes in the vicinity of 1 . It can be determined that:

$f(0)=-5.1, f(1)=4.4, f(-1)=-2.2$. Searching for a root between 0 and 1 results in finding.

$r=0.746$ after 11 iterations. Dividing $f(x)$ by $(x-0.746)$ yields (with three decimal place accuracy):

$$
f(x)=(x-0.746) f_{1}(x)
$$

where:

$$
f_{1}(x)=x^{5}+1.546 x^{4}+3.253 x^{3}+0.927 x^{2}+3.791 x+6.828
$$

It can be seen that for $f_{1}(x),\left|\alpha_{1}\right|=1.546>1$. Following the above procedure:

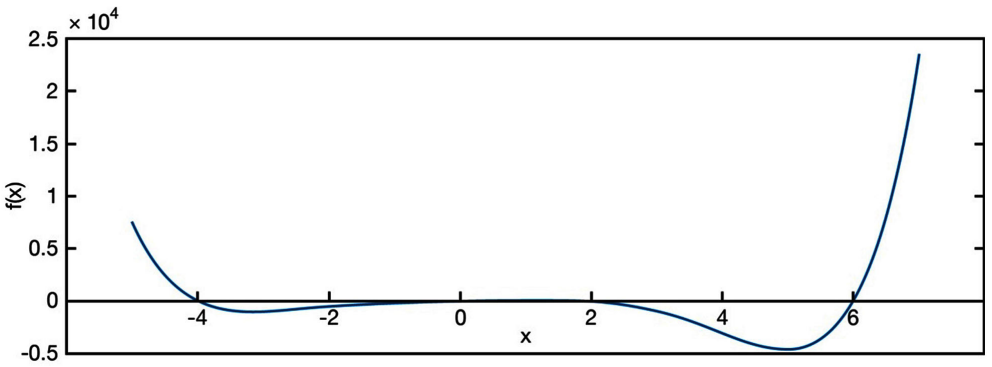

Figure 2. A graph representing $f(x)$ of example 2 . 


$$
\begin{gathered}
k_{2}=-10.62 \\
f_{1}^{\prime}(x)=5 x^{4}+6.184 x^{3}+9.759 x^{2}+1.854 x+3.791 \\
f_{1}^{\prime}(-1.546)=29.96=m_{2} \\
x_{3}=-1.546 \text {, and } f_{1}\left(x_{3}\right)=y_{3}=-8.837, \\
x_{4}=-1.546+(8.837 / 29.96)=-1.25 \text {, and } f_{1}\left(x_{4}\right)=-2 \text {, and } f_{1}(-1)=1.257 .
\end{gathered}
$$

A real root of $r=-1.108$ can be found between -1 , and -1.25 with three decimal place accuracy after 10 iterations. Therefore $f(x)$ has two real roots so far with magnitudes in the vicinity of $|r|=1$. By factorizing $f_{1}(x)$ :

$$
f_{1}(x)=(x+1.108) f_{2}(x)
$$

where:

$$
f_{2}(x)=x^{4}+0.438 x^{3}+2.768 x^{2}-2.14 x+6.162
$$

To find the roots of $f_{2}(x)$, the procedures described above for quartic equations can be used. By using method $\# 2$, the roots of the derivative function $f_{2}^{\prime}(x)$ need to be found. The real root of $f_{2}^{\prime}(x)$ is found after 7 iterations at $x$ $=0.333$ by using the method in [10]. However, it can be seen that $f_{2}(x)$ at this minimum point is positive (i.e. at 5.787). Therefore, it can be determined from

\begin{tabular}{|c|c|}
\hline Condition to Check & Result \\
\hline If $g(x)$ has one real root & $f(x)$ may have one minimum at that point \\
\hline If $g(x)$ has three real roots & $f(x)$ has two minimums and one maximum point \\
\hline If $\alpha_{4}<0$ & $f(x)$ has real roots \\
\hline If $\alpha_{4}=0$ & $f(x)$ has a real root at $x=0$ \\
\hline If $\alpha_{4}>0$ & $f(x)$ may or may not have real roots \\
\hline $\begin{array}{l}\text { If } g(x) \text { has one real root repeated three times, and } \\
\text { at that same point } f(x) \text { is also zero }\end{array}$ & that root is a real root of $f(x)$ repeated four times \\
\hline $\begin{array}{l}\text { If } g(x) \text { has one real root and at that same point } \\
f(x) \text { is also zero }\end{array}$ & that root is a repeated real root of $f(x)$ \\
\hline $\begin{array}{l}\text { If } f(x) \text { is not zero or negative at any minimum } \\
\text { point }\end{array}$ & the quartic equation has no real root \\
\hline $\begin{array}{l}\text { If } g(x) \text { has one real root and } f(x) \text { is zero or } \\
\text { negative at the minimum point }\end{array}$ & $\begin{array}{l}f(x) \text { has two real roots (or one repeated real root) } \\
\text { and two complex conjugate roots }\end{array}$ \\
\hline $\begin{array}{l}\text { If } g(x) \text { has three real roots, and } f(x) \text { is negative at } \\
\text { two minimum points and is not negative at the } \\
\text { maximum point }\end{array}$ & all the roots of the quartic equation are real \\
\hline
\end{tabular}
Table 1 that $f_{2}(x)$ has no real roots. Therefore, method \#1 should be used to find the roots of $f_{2}(x)$ which are all complex.

Following the procedure of method $\# 1$ for quartic equations to find the roots of $f_{2}(x)$ :

$$
x=z-0.438 / 4=z-0.1095
$$

Table 1. A list of conditions to check the type of the roots of a quartic equation. 
Therefore:

$$
f_{2}(z)=z^{4}+a z^{2}+b z+c
$$

where:

$$
a=2.696, b=-2.7356, c=6.429
$$

If $t=\gamma^{2}=\left(R_{1}+R_{2}\right)^{2}$, where $R_{1}$ and $R_{2}$ are two roots of $f_{2}(z)$, the following cubic equation will result:

$$
f(t)=t^{3}+5.392 t^{2}-18.447 t-7.4835
$$

A real root of $f(t)$ is found by using the procedure in [10] at $t_{1}=2.646$ (with three decimal place accuracy) after 10 iterations.

Therefore:

$$
\sqrt{t_{1}}= \pm 1.6266
$$

For $R_{1}+R_{2}=1.6266$.

If $R_{1} R_{2}=\lambda$, by using Equation (11):

$$
\lambda^{2}-5.342 \lambda+6.429=0
$$

The roots of this $2^{\text {nd }}$ order equation are at $\lambda=1.831$ and $\lambda=3.51$.

Then:

$$
\begin{aligned}
& R_{1}+R_{2}=1.6266 \text { and } R_{1} R_{2}=1.831 \text { yield: } \\
& \qquad R_{1}, R_{2}=0.8133 \pm 1.08145 i
\end{aligned}
$$

Then by using Equation (12):

$$
z^{2}+1.6266 z+3.51=0
$$

$R_{3}$ and $R_{4}$ are the roots of this equation as:

$$
R_{3}, R_{4}=-0.8133 \pm 1.68776 i
$$

The roots of $f_{2}(x)$ can be found by subtracting 0.1095 from the roots of $f_{2}(z)$ at $0.7038 \pm 1.08145 i$, and at $-0.9228 \pm 1.68776 i$. Therefore the roots of $f(x)$ are found at: $-1.108,0.746,-0.9228 \pm 1.68776 i$, and $0.7038 \pm 1.08145 i$.

Figure 3 shows a graphical representation of $f(x)$.

\section{Discussion}

Two methods for solving quartic equations that use a recently presented technique for solving cubic equations [10] are described. The $1^{\text {st }}$ technique that combines the method in [10] with a technique originally developed by Descartes [16] can be used to solve quartic equations regardless of whether the roots are real or complex. The second presented method offers an efficient technique to solve quartic equations with real roots. The paper further describes a general technique for solving higher order polynomial equations and providing all the real roots of those equations. Each of the methods discussed in this paper combines the use of algebraic equations with some iterative procedures. The methods rely on initially establishing reasonable boundaries for the individual roots and unlike many previous techniques, none of them requires the use of complicated 


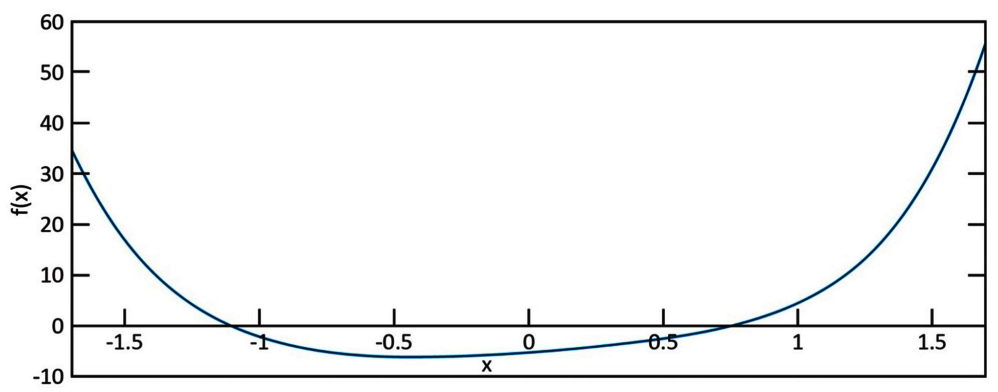

Figure 3. A graph representing $f(x)$ of example 3.

formulas, roots of complex numbers, or graphs. The examples presented show how the methods are applied and their efficiencies.

\section{Conclusions}

A number of methods have been presented in the literature and are used in practice to find the roots of polynomial equations with real coefficients. A new technique was recently presented to find the roots of cubic functions [10] which is simple and yet efficient enough to provide the roots of cubic equations within a short period of time even by hand calculation. This paper describes two methods for solving quartic equations and a more general technique for solving polynomial equations of degree 5 or higher. Unlike previous methods, none of the methods presented requires finding the roots of complex numbers, or utilizing complicated formulas and procedures or graphs. In these new techniques, emphasis is placed on initially finding the vicinities of the individual real roots and then finding those roots efficiently by using algebraic and/or iterative procedures.

The next phase of this research can focus on further investigation of the efficiency of the presented method for solving high order polynomial equations when some of the coefficients are much larger/smaller than other coefficients.

\section{Conflicts of Interest}

The author declares no conflicts of interest regarding the publication of this paper.

\section{References}

[1] Atkinson, K.E. (1989) An Introduction to Numerical Analysis. John Wiley \& Sons, Inc., Hoboken.

[2] Scavo, T.R. and Thoo, J.B. (1995) On the Geometry of Halley's Method. The American Mathematical Monthly, 102, 417-426. https://doi.org/10.1080/00029890.1995.12004594

[3] Akritas, A.G., Strzebonski, A.W. and Vigklas, P.S. (2008) Improving the Performance of the Continued Fractions Method Using New Bounds of Positive Roots. Nonlinear Analysis: Modelling and Control, 13, 265-279. https://doi.org/10.15388/NA.2008.13.3.14557

[4] Collins, G.E. (2001) Polynomial Minimum Root Separation. Journal of Symbolic 
Computation, 32, 467-473. https://doi.org/10.1006/jsco.2001.0481

[5] Edelman, A. and Kostlan, E. (1995) How Many Zeros of a Random Polynomial Are Real? Bulletin of the American Mathematical Society, 32, 1-37. https://doi.org/10.1090/S0273-0979-1995-00571-9

[6] Hirst, H.P. and Macey, W.T. (1997) Bounding the Roots of Polynomials. The College Mathematics Journal, 28, 292-295. https://doi.org/10.2307/2687152

[7] Sun, Y.J. and Hsieh, J.G. (1996) A Note on the Circular Bound of Polynomial Zeros. IEEE Transactions on Circuits and Systems, 43, 476-478. https://doi.org/10.1109/81.503258

[8] Dalal, A. and Govil, N.K. (2017) On Comparison of Annuli Containing All the Zeros of a Polynomial. Applicable Analysis and Discrete Mathematics, 11, 232-241. https://doi.org/10.2298/AADM1701232D

[9] Gao, L. and Govil, N.K. (2018) Annular Bounds for the Zeros of a Polynomial. International Journal of Mathematics and Mathematical Sciences, 2018, Article ID: 6047387. https://doi.org/10.1155/2018/6047387

[10] Tehrani, F.T. (2016) A Simple Approach to Solving Cubic Equations. The Mathematical Gazette, 100, 225-232. https://doi.org/10.1017/mag.2016.58

[11] Aude, H.T.R. (1949) Notes on Quartic Curves. The American Mathematical Monthly, 56, 165-170. https://doi.org/10.1080/00029890.1949.11999352

[12] Faucette, W.M. (1996) A Geometric Interpretation of the Solution of the General Quartic Polynomial. The American Mathematical Monthly, 103, 51-57. https://doi.org/10.2307/2975214

[13] Kappe, L.C. and Warren, B. (1989) An Elementary Test for the Galois Group of a Quartic Polynomial. The American Mathematical Monthly, 96, 133-137. https://doi.org/10.1080/00029890.1989.11972158

[14] Rees, E.L. (1922) Graphical Discussion of the Roots of a Quartic Equation. The American Mathematical Monthly, 29, 51-55. https://doi.org/10.1080/00029890.1922.11986100

[15] Shmakov, S.L. (2011) A Universal Method of Solving Quartic Equations. International Journal of Pure and Applied Mathematics, 71, 251-259.

[16] Froberg, C.E. (1965) Introduction to Numerical Analysis. Addison-Wesley Publishing Company Inc., Reading.

[17] Thomas, J.M. (1941) Sturm's Theorem for Multiple Roots. National Mathematics Magazine, 15, 391-394. https://doi.org/10.2307/3028551

[18] Shamseddine, K. and Berz, M. (2010) Analysis of the Levi-Civita Field, a Brief Overview. In: Berz, M. and Shamseddine, K., Eds., Contemporary Mathematics, Advances in p-Adic and Non-Archimedean Analysis, Vol. 508, The American Mathematical Society, Providence, 215-237. https://doi.org/10.1090/conm/508/10002 